\title{
On covariant nonlocal chiral quark models with separable interactions
}

\author{
D. Gómez Dumm ${ }^{a, b}$, A.G. Grunfeld ${ }^{c}$ and N.N. Scoccola ${ }^{b, c, d}$ \\ ${ }^{a}$ IFLP - Dpto. de Fúsica, Universidad Nacional de La Plata, C.C. 67, 1900 La Plata, Argentina \\ ${ }^{b}$ CONICET, Rivadavia 1917, 1033 Buenos Aires, Argentina \\ ${ }^{c}$ Physics Department, Comisión Nacional de Energía Atómica, \\ Av. Libertador 8250, 1429 Buenos Aires, Argentina \\ d Universidad Favaloro, Solís 453, 1078 Buenos Aires, Argentina
}

\begin{abstract}
We present a comparative analysis of chiral quark models which include nonlocal covariant fourfermion couplings. We consider two alternative ways of introducing the nonlocality, as well as various shapes for the momentum-dependent form factors governing the effective interactions. In all cases we study the behavior of model parameters and analyze numerical results for constituent quark masses and quark propagator poles. Advantages of these covariant nonlocal schemes over instantaneous nonlocal schemes and the standard NJL model are pointed out.
\end{abstract}

PACS numbers: 12.39.Ki, 11.30.Rd, 11.10.Lm

\section{INTRODUCTION}

The description of strong interactions in the nonperturbative regime is still one of the most important open problems in particle physics. It is believed that the strong interaction Lagrangian supports an $\mathrm{SU}(2)$ chiral symmetry which is dynamically broken at low energies, and pions play the rôle of the corresponding Goldstone bosons. A simple scheme including these properties is the well known Nambu-Jona-Lasinio (NJL) model [1], proposed more than four decades ago. The NJL model has been widely used as an schematic effective theory for QCD [2, 3, 4], allowing e.g. the description of light mesons as fermion-antifermion composite states.

In the NJL model quarks interact through a local, chiral invariant four fermion coupling. Due to the local nature of this interaction, the corresponding Schwinger-Dyson and Bethe-Salpeter equations become relatively simplified. However, the main drawbacks of the model are direct consequences of this locality: loop integrals are divergent (and therefore have to be regulated somehow), and the model is nonconfining. The absence of confinement is basically related to the fact that the dynamically generated constituent quark masses are momentum independent. This imposes a severe restriction on the range of applicability of the model, since a $\bar{q} q$ continuum opens up at energies of twice the constituent mass. Since the NJL model is nonrenormalizable, in practice it is necessary to apply some form of ultraviolet regularization, with the introduction of a finite cutoff parameter. The adopted regularization scheme has to be regarded as a part of the model itself. A variety of schemes have been used in the literature, such as sharp three- or four-momentum cutoffs, and proper time or Pauli-Villars regulators. Although the model does contain regularization-independent information [5], and results obtained within various regularization schemes are found to be qualitatively similar [ $\underline{6}$, the choice of any particular scheme lacks a strong physical motivation. Moreover, it can be seen that one needs further regularization prescriptions in order to carry out calculations beyond the leading order in the $1 / N_{c}$ expansion (a new cutoff is required for meson loops [] $)$. On the other hand, the introduction of a finite cutoff is somewhat problematic in the anomalous sector: if low energy theorems for anomalous processes such as $\pi^{0} \rightarrow 2 \gamma$ are assumed to be valid, then the corresponding quark loop integrals should include a complete set of quark states. In the NJL framework, this can be achieved either by leaving the anomalous diagrams ad-hoc unregulated [8] or by including additional terms in the Lagrangian in order to recover the anomalous Ward identities [5].

As a way to improve upon the NJL model, extensions which include nonlocal interactions have been proposed (see Ref. 9] and references therein). In fact, nonlocality arises naturally in the context of several well established approaches to low energy quark dynamics, as e.g. the instanton liquid model [10] and the Schwinger-Dyson resummation techniques 11. Lattice QCD calculations 12] also indicate that quark interactions should act over a certain range in the momentum space. Moreover, it has been argued that nonlocal extensions of the NJL model do not show some of the above mentioned inconveniences of the local theory. Indeed, nonlocal interactions regularize the model in such a way that anomalies are preserved [13] and charges are properly quantized, the effective interaction is finite to all orders in the loop expansion and therefore there is not need to introduce extra cutoffs [14], soft regulators such as Gaussian functions lead to small next-to-leading order corrections [15], etc. In addition, it has been shown [16, 17] that a proper choice of the nonlocal form factor and the model parameters can lead to some form of quark confinement, in the sense that the effective quark propagator has no poles at real energies 18$]$.

In order to bring the problem into a tractable form, most of the calculations reported in the literature deal with nonlocal interactions which are separable in momentum space. In fact, basically two alternative schemes to introduce nonlocality in a separable way have been considered. One of them [16, 17, 19] is inspired on the instanton liquid picture 
of QCD, which indeed gives rise to an effective separable nonlocal four quark vertex. The other one [20, 21] might be understood as a separable approximation to an effective one-gluon exchange interaction. Considerable work has been done using one scheme or the other. This includes studies of the mesonic [16, 17, 22, 23] and the baryonic [24, 25] sectors, as well as the behavior of quark matter at finite temperature and densities [26]. The aim of the present work is to present a detailed comparison of both approaches and their relation with the NJL model, analyzing the respective input parameter ranges for different form factors and discussing the validity of low energy theorems.

The article is organized as follows. In Sec. II we introduce a common framework to deal with the above mentioned approaches to nonlocal separable interactions. Analytical expressions for mean field quantities, meson masses and decay constants are given for both schemes. In Sec. III, the validity of various chiral relations is explicitly shown. In Sec. IV we quote some numerical results for definite nonlocal form factors. We consider here the widely used Gaussian form factor, as well as a family of Lorentzian functions which allow us to interpolate between soft form factors and sharp NJL-like cutoffs. Finally, our main results and conclusions are outlined in Sec. V.

\section{COVARIANT NONLOCAL MODELS WITH SEPARABLE INTERACTIONS}

\section{A. Effective action}

Let us begin by stating the Euclidean action for the nonlocal chiral quark model in the case of two light flavors,

$$
S_{E}=\int d^{4} x\left\{\bar{\psi}(x)\left(-i \not \partial+m_{c}\right) \psi(x)-\frac{G}{2} j_{a}(x) j_{a}(x)\right\} .
$$

Here $m_{c}$ is the current quark mass, which is assumed to be equal for $u$ and $d$ quarks. As mentioned in the Introduction, separable nonlocal interactions might be introduced in two alternative ways. In what follows, we will call them "Scheme I" and "Scheme II". These schemes are characterized by the form of the nonlocal currents $j_{a}(x)$ in Eq. (1). In the case of Scheme I [16, 17, 19], the effective interactions are based in an instanton liquid picture of QCD. The currents can be written as

$$
j_{a}(x)=\int d^{4} y d^{4} z r(y-x) r(x-z) \bar{\psi}(y) \Gamma_{a} \psi(z)
$$

where we have defined $\Gamma_{a}=\left(\mathbb{1}, i \gamma_{5} \vec{\tau}\right), \tau_{i}$ being the Pauli matrices acting on flavor space.

On the other hand, Scheme II [20, 21] arises from a separable form of the effective one-gluon exchange picture. In this case the nonlocal currents $j_{a}(x)$ are given by

$$
j_{a}(x)=\int d^{4} z g(z) \bar{\psi}\left(x+\frac{z}{2}\right) \Gamma_{a} \psi\left(x-\frac{z}{2}\right) .
$$

The functions $r(x-y)$ and $g(z)$ in Eqs. (2) and (3), respectively, are nonlocal form factors characterizing the corresponding interactions. It is convenient to translate them into momentum space. Since Lorentz invariance implies that they can only be functions of $p^{2}$, we will use for the Fourier transforms of these regulators the forms $r\left(p^{2}\right)$ and $g\left(p^{2}\right)$ from now on. A schematic representation of the four quark interactions in both schemes is given in Fig. 1 .

In order to deal with meson degrees of freedom, one can perform a standard bosonization of the theory. This is done by considering the corresponding partition function $Z=\int \mathcal{D} \bar{\psi} \mathcal{D} \psi \exp \left[-S_{E}\right]$, and introducing auxiliary fields $S_{a}(x)=(\sigma(x), \vec{\pi}(x))$, where $\sigma(x)$ and $\vec{\pi}(x)$ are scalar and pseudoscalar mesons, respectively. Integrating out the quark fields we get

$$
Z=\int \mathcal{D} \sigma \mathcal{D} \vec{\pi} \exp \left[-S_{E}^{\mathrm{bos}}\right]
$$

where

$$
S_{E}^{\mathrm{bos}}=-\ln \operatorname{det} A+\frac{1}{2 G} \int \frac{d^{4} p}{(2 \pi)^{4}} S_{a}(p) S_{a}(-p)
$$

The operator $A$ reads, in momentum space,

$$
A\left(p, p^{\prime}\right)=\left(-\not p+m_{c}\right)(2 \pi)^{4} \delta^{(4)}\left(p-p^{\prime}\right)+h\left(p, p^{\prime}\right) \Gamma_{a} S_{a}\left(p-p^{\prime}\right)
$$



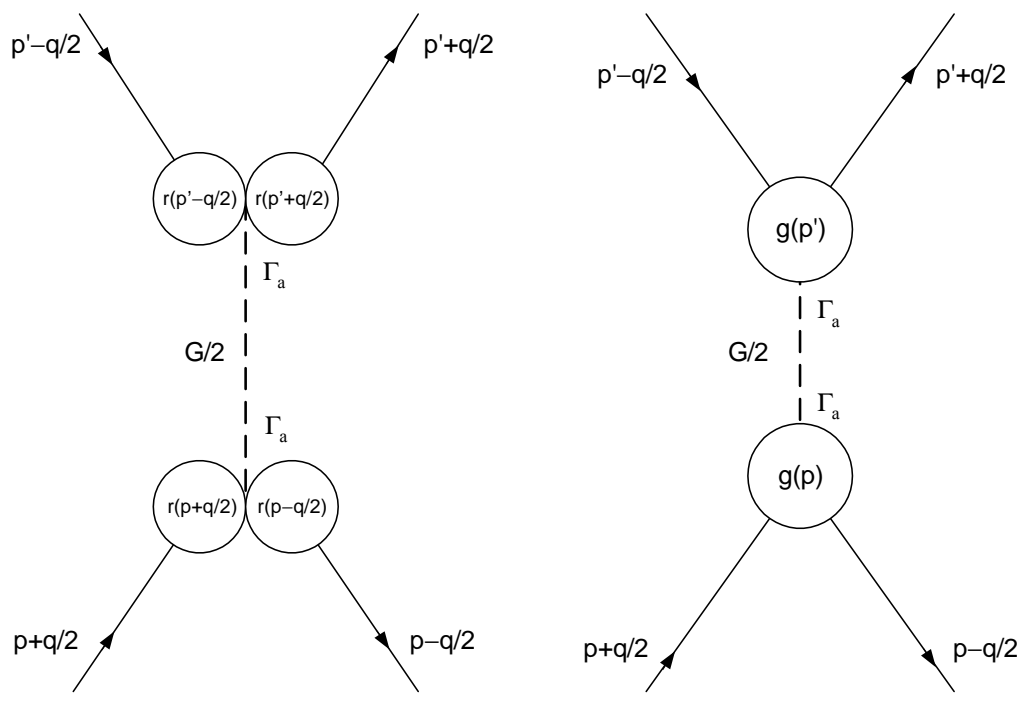

FIG. 1: Schematic representation of the four fermion interaction in Scheme I (left) and Scheme II (right).

where

$$
h\left(p, p^{\prime}\right)= \begin{cases}r\left(p^{2}\right) r\left(p^{2}\right) & \text { (Scheme I) } \\ g\left[\left(\frac{p+p^{\prime}}{2}\right)^{2}\right] & \text { (Scheme II) } .\end{cases}
$$

At this stage we assume that the $\sigma$ field has a nontrivial translational invariant mean field value $\bar{\sigma}$, while the mean field values of the pseudoscalar fields $\pi_{i}$ are zero. Thus we write

$$
\begin{aligned}
& \sigma(x)=\bar{\sigma}+\delta \sigma(x) \\
& \vec{\pi}(x)=\delta \vec{\pi}(x)
\end{aligned}
$$

Replacing in the bosonized effective action, and expanding in powers of the meson fluctuations, we get

$$
S_{E}^{\text {bos }}=S_{E}^{\mathrm{MFA}}+S_{E}^{\mathrm{quad}}+\ldots
$$

Here the mean field action per unit volume reads

$$
\frac{S_{E}^{\mathrm{MFA}}}{V^{(4)}}=-4 N_{c} \int \frac{d^{4} p}{(2 \pi)^{4}} \ln \left[p^{2}+\Sigma^{2}(p)\right]+\frac{\bar{\sigma}^{2}}{2 G}
$$

where $\Sigma(p)=m_{c}+h(p, p) \bar{\sigma}$. The quadratic terms are given by

$$
S_{E}^{\text {quad }}=\frac{1}{2} \int \frac{d^{4} p}{(2 \pi)^{4}}\left[G^{+}\left(p^{2}\right) \delta \sigma(p) \delta \sigma(-p)+G^{-}\left(p^{2}\right) \delta \vec{\pi}(p) \cdot \delta \vec{\pi}(-p)\right],
$$

where

$$
G^{ \pm}\left(p^{2}\right)=\frac{1}{G}-8 N_{c} \int \frac{d^{4} q}{(2 \pi)^{4}} h^{2}\left(q^{+}, q^{-}\right) \frac{\left[\left(q^{+} \cdot q^{-}\right) \mp \Sigma\left(q^{+}\right) \Sigma\left(q^{-}\right)\right]}{\left[\left(q^{+}\right)^{2}+\Sigma^{2}\left(q^{+}\right)\right]\left[\left(q^{-}\right)^{2}+\Sigma^{2}\left(q^{-}\right)\right]}
$$

with $q^{ \pm}=q \pm p / 2$.

\section{B. Mean field approximation and chiral condensates}

In order to find the mean field values $\bar{\sigma}$, one has to minimize the action $S_{E}^{\mathrm{MFA}}$. A straightforward exercise leads to the gap equation

$$
\bar{\sigma}-8 N_{c} G \int \frac{d^{4} p}{(2 \pi)^{4}} h(p, p) \frac{\Sigma(p)}{p^{2}+\Sigma^{2}(p)}=0 .
$$


Now the chiral condensates are given by the vacuum expectation values $\langle\bar{q} q\rangle=\langle\bar{u} u\rangle=\langle\bar{d} d\rangle$. They can be easily obtained by performing the variation of $Z^{\mathrm{MFA}}=\exp \left[-S_{E}^{M F A}\right]$ with respect to the corresponding current quark masses. We obtain

$$
\langle\bar{q} q\rangle=-4 N_{c} \int \frac{d^{4} p}{(2 \pi)^{4}} \frac{\Sigma(p)}{p^{2}+\Sigma^{2}(p)}
$$

\section{Meson masses and quark-meson coupling constants}

In what follows we consider in particular the case of pseudoscalar mesons. The expressions for scalar mesons are completely equivalent, just replacing the upper index "-" by "+" where it corresponds.

The pion mass can be obtained by solving the equation

$$
G^{-}\left(-m_{\pi}^{2}\right)=0
$$

Now, one has to perform a wave function renormalization of the pseudoscalar fields. The renormalized pion field $\overrightarrow{\tilde{\pi}}(p)=\vec{\pi}(p) / Z_{\pi}^{1 / 2}$ is defined by fixing the residue of $G^{-}\left(p^{2}\right)$ at the pion pole, i.e. by demanding that in the vicinity of the pole the corresponding contribution to the quadratic lagrangian is given by

$$
\left(\mathcal{L}_{E}^{\text {quad }}\right)_{\pi}=\frac{1}{2}\left(p^{2}+m_{\pi}^{2}\right) \overrightarrow{\tilde{\pi}}(p) \cdot \overrightarrow{\tilde{\pi}}(-p)
$$

In this way, one obtains

$$
Z_{\pi}^{-1}=\left.\frac{d G^{-}(p)}{d p^{2}}\right|_{p^{2}=-m_{\pi}^{2}}
$$

Finally the $\pi q \bar{q}$ coupling constant $G_{\pi q \bar{q}}$ is given by the residue of the pion propagator at the pole, leading to

$$
G_{\pi q \bar{q}}=Z_{\pi}^{1 / 2}
$$

\section{Pion weak decay constant}

By definition the pion weak decay constant $f_{\pi}$ is given by the matrix element of the axial current $A_{\mu}^{a}(x)$ between the vacuum and the renormalized one-pion state at the pion pole:

$$
\left\langle 0\left|A_{\mu}^{a}(0)\right| \tilde{\pi}^{b}(p)\right\rangle=i \delta^{a b} p_{\mu} f_{\pi} .
$$

In order to obtain an explicitly expression for the axial current, we have to "gauge" the effective action $S_{E}$ by introducing a set of axial gauge fields $\mathcal{A}_{\mu}^{a}(x)$. For a local theory this "gauging" procedure is usually done by performing the replacement

$$
\partial_{\mu} \rightarrow \partial_{\mu}+\frac{i}{2} \gamma_{5} \vec{\tau} \cdot \overrightarrow{\mathcal{A}}_{\mu}(x)
$$

In the present case - owing to the nonlocality of the involved fields - one has to perform an additional replacement, namely

$$
\gamma_{0} \Gamma_{a} S_{a}(x) \rightarrow \begin{cases}W_{A}(y, x) \gamma_{0} \Gamma_{a} S_{a}(x) W_{A}(x, z) & \text { (Scheme I) } \\ W_{A}(x+z / 2, x) \gamma_{0} \Gamma_{a} S_{a}(x) W_{A}(x, x-z / 2) & \text { (Scheme II) }\end{cases}
$$

Here $y$ and $z$ are the integration variables in the definitions of the nonlocal currents (see Eqs. (2) and (3)), and the function $W_{A}(x, y)$ is defined by

$$
W_{A}(x, y)=\mathrm{P} \exp \left[\frac{i}{2} \int_{x}^{y} d s_{\mu} \gamma_{5} \vec{\tau} \cdot \overrightarrow{\mathcal{A}}_{\mu}(s)\right]
$$

where $s$ runs over an arbitrary path connecting $x$ with $y$. This corresponds to the introduction of a parallel transport [27]. 
Once the gauged effective action is built, it is easy to get the axial current as the derivative of this action with respect to $\mathcal{A}_{\mu}^{a}(x)$, evaluated at $\overrightarrow{\mathcal{A}}_{\mu}(x)=0$. Performing the derivative of the resulting expressions with respect to the renormalized meson fields, we can finally identify the corresponding meson weak decay constants. After a rather lengthy calculation we obtain

$$
f_{\pi}=Z_{\pi}^{1 / 2} \frac{F\left(-m_{\pi}^{2}\right)-F(0)}{m_{\pi}^{2}}
$$

where $F\left(p^{2}\right)$ is given by

$$
F\left(p^{2}\right)=m_{c} J\left(p^{2}\right)+\bar{\sigma} K\left(p^{2}\right)
$$

with

$$
\begin{aligned}
J\left(p^{2}\right) & =8 N_{c} \int \frac{d^{4} q}{(2 \pi)^{4}} h\left(q^{+}, q^{-}\right) \frac{\left[\left(q^{+} \cdot q^{-}\right)+\Sigma\left(q^{+}\right) \Sigma\left(q^{-}\right)\right]}{\left[\left(q^{+}\right)^{2}+\Sigma^{2}\left(q^{+}\right)\right]\left[\left(q^{-}\right)^{2}+\Sigma^{2}\left(q^{-}\right)\right]} \\
K\left(p^{2}\right) & =8 N_{c} \int \frac{d^{4} q}{(2 \pi)^{4}} h^{2}\left(q^{+}, q^{-}\right) \frac{\left[\left(q^{+} \cdot q^{-}\right)+\Sigma\left(q^{+}\right) \Sigma\left(q^{-}\right)\right]}{\left[\left(q^{+}\right)^{2}+\Sigma^{2}\left(q^{+}\right)\right]\left[\left(q^{-}\right)^{2}+\Sigma^{2}\left(q^{-}\right)\right]}
\end{aligned}
$$

(here, as before, $q^{ \pm}=q \pm p / 2$ ).

Notice that in terms of the functions $J\left(p^{2}\right)$ and $K\left(p^{2}\right)$ Eqs. (14) and (16) can be written as

$$
\begin{aligned}
F(0) & =\bar{\sigma} / G \\
K\left(-m_{\pi}^{2}\right) & =1 / G .
\end{aligned}
$$

Replacing these expressions in Eq. (24), we get the relation

$$
\begin{gathered}
m_{\pi}^{2} f_{\pi}=m_{c} Z_{\pi}^{1 / 2} J\left(-m_{\pi}^{2}\right) . \\
\text { E. } \quad \pi^{0} \rightarrow \gamma \gamma \text { decay }
\end{gathered}
$$

Let us analyze in our context the anomalous decay of the $\pi^{0}$ meson into two photons. In general, the amplitude for this process can be written as

$$
A\left(\pi^{0} \rightarrow \gamma \gamma\right)=4 \pi \alpha g_{\pi \gamma \gamma} \epsilon_{\mu \nu \alpha \beta} \varepsilon_{1}^{* \mu} \varepsilon_{2}^{* \nu} k_{1}^{\alpha} k_{2}^{\beta}
$$

where $\alpha$ is the fine structure constant and $k_{i}, \varepsilon_{i}$ stand for the momenta and polarizations of the outgoing photons respectively. The partial decay width is then given by

$$
\Gamma\left(\pi^{0} \rightarrow \gamma \gamma\right)=\frac{\pi}{4} \alpha^{2} m_{\pi^{0}}^{3} g_{\pi \gamma \gamma}^{2}
$$

The coefficients $g_{\pi \gamma \gamma}$ are given by quark loop integrals. Besides the usual "triangle" diagram, given by a closed quark loop with one pion and two photon vertices, in the present nonlocal schemes one has a second contribution [17] in which one of the quark-photon vertices arises from the gauge fields entering the nonlocal currents [see Eq. (22)]. The sum of both contributions leads to

$$
g_{\pi \gamma \gamma}=I\left(-m_{\pi}^{2}\right) Z_{\pi}^{1 / 2}
$$

where

$$
\begin{aligned}
I\left(-m_{\pi}^{2}\right)= & \frac{8}{3} N_{c} \int \frac{d^{4} q}{(2 \pi)^{4}} \frac{h\left(q+k_{1}, q-k_{2}\right)}{\left[q^{2}+\Sigma^{2}(q)\right]\left[\left(q+k_{1}\right)^{2}+\Sigma^{2}\left(q+k_{1}\right)\right]\left[\left(q-k_{2}\right)^{2}+\Sigma^{2}\left(q-k_{2}\right)\right]} \\
& \times\left\{\Sigma(q)+\frac{q^{2}}{2}\left[\frac{\left[\Sigma\left(q-k_{2}\right)-\Sigma(q)\right]}{\left(k_{2} \cdot q\right)}-\frac{\left[\Sigma\left(q+k_{1}\right)-\Sigma(q)\right]}{\left(k_{1} \cdot q\right)}\right]\right\} .
\end{aligned}
$$

Notice that for on-shell photons the above integral is a function of the scalar product $\left(k_{1} \cdot k_{2}\right)$ only, and in Euclidean space one has $\left(k_{1} \cdot k_{2}\right)=-m_{\pi}^{2} / 2$. 


\section{CHIRAL RELATIONS}

We start by discussing the Goldberger-Treiman (GT) relation, which states that in the chiral limit $\left(m_{c} \rightarrow 0\right)$ one has

$$
f_{\pi, 0} G_{\pi q \bar{q}, 0}=\bar{\sigma}_{0}
$$

(here, and in the following, subindices 0 mean that quantities are evaluated in the chiral limit). In our case, from Eqs. (24) and (25) one has

$$
f_{\pi, 0}=Z_{\pi, 0}^{1 / 2}\left[\frac{F_{0}\left(-p^{2}\right)-F_{0}(0)}{p^{2}}\right]_{p^{2}=0}=-\bar{\sigma}_{0} Z_{\pi, 0}^{1 / 2} K_{0}^{\prime}(0)
$$

where prime denotes derivation with respect to $p^{2}$. Now, an explicit calculation shows that $f_{\pi, 0}$ can be written in terms of the momentum-dependent constituent masses $\Sigma(q)$ as

$$
f_{\pi, 0}^{2}=2 N_{c} \int \frac{d^{4} q}{(2 \pi)^{4}} \frac{2 \Sigma_{0}^{2}(q)-q^{2} \Sigma_{0}(q) \Sigma_{0}^{\prime}(q)}{\left[q^{2}+\Sigma_{0}^{2}(q)\right]^{2}} .
$$

Then, taking into account Eqs. (18) and (19), and noting that $G_{0}^{-}\left(p^{2}\right)=1 / G-K_{0}\left(p^{2}\right)$, one gets

$$
K_{0}^{\prime}(0)=-Z_{\pi, 0}^{-1}=-G_{\pi q \bar{q}, 0}^{-2} .
$$

The GT relation (33) follows immediately from (34) and (36).

Next, let us consider the Gell-Mann-Oakes-Renner (GOR) relation, which can be obtained using Eq. (28). By performing a chiral expansion, and keeping only the lowest nonzero order at both sides of this equation, one has

$$
m_{\pi}^{2} f_{\pi, 0}=m_{c} Z_{\pi, 0}^{1 / 2} J_{0}(0) .
$$

In addition, from Eq. (15) it is easy to see that

$$
\langle\bar{u} u+\bar{d} d\rangle_{0}=2\langle\bar{q} q\rangle_{0}=-\bar{\sigma}_{0} J_{0}(0)
$$

thus using the GT relation one arrives to

$$
m_{c}\langle\bar{u} u+\bar{d} d\rangle_{0}=-f_{\pi, 0}^{2} m_{\pi}^{2},
$$

which is the form taken by the GOR relation in the isospin limit.

Finally, we study the anomalous effective coupling $g_{\pi \gamma \gamma}$ in the chiral limit. Considering Eq. (32), one can perform an expansion in powers of the momenta $k_{1}$ and $k_{2}$ and take the limit $m_{\pi}^{2} \rightarrow 0$. This leads to

$$
I_{0}(0)=\frac{N_{c}}{12 \pi^{2} \bar{\sigma}_{0}} .
$$

Now, for $N_{c}=3$, using the GT relation one gets

$$
g_{\pi \gamma \gamma, 0}=\frac{1}{4 \pi^{2} f_{\pi, 0}},
$$

which is the expected result according to low energy theorems and Chiral Perturbation Theory.

We stress that the derivation of the above chiral relations holds for both schemes I and II.

\section{COMPARATIVE ANALYSIS FOR DEFINITE FORM FACTORS}

In what follows we consider two types of form factors which have been frequently used in the literature. One is the Gaussian form factor,

$$
g_{\mathrm{G}}\left(p^{2}\right)=\left[r_{\mathrm{G}}\left(p^{2}\right)\right]^{2}=\exp \left(-p^{2} / \Lambda^{2}\right)
$$


The other is the Lorenztian form factor

$$
g_{\mathrm{Ln}}\left(p^{2}\right)=\left[r_{\operatorname{Ln}}\left(p^{2}\right)\right]^{2}=\frac{1}{1+\left(p^{2} / \Lambda^{2}\right)^{n}},
$$

where $n \geq 2$ is an integer that allows us to interpolate between soft form factors (such as the Gaussian one) and sharp form factors as e.g. a step function $g_{\text {Step }}\left(p^{2}\right)=\Theta\left(\Lambda^{2} / p^{2}-1\right)$.

With the choice $g\left(p^{2}\right)=\left[r\left(p^{2}\right)\right]^{2}$, both schemes I and II lead to the same expressions for the mean field quantities. Moreover, in the chiral limit, the expressions for the pion decay constant converge in both cases to the result in Eq. (35). This simplifies the comparison of the present type of nonlocal models with other frameworks such as instantaneous nonlocal schemes and the usual NJL model. In the following subsection we will present a comparative analysis, considering in particular the chiral limit. Results corresponding to the case of finite quark masses will be discussed in subsection B.

\section{A. Chiral limit case}

In this limit the pion is massless. Moreover, a simple dimensional analysis shows that, for the kind of models under study, the chiral condensate can be expressed as

$$
\left[-\langle\bar{q} q\rangle_{0}\right]^{1 / 3}=f_{\pi, 0} \mathcal{F}\left(G \Lambda^{2}\right) .
$$

Thus, for a given value of $f_{\pi, 0}$ the chiral condensate only depends on a single dimensionless parameter, $G \Lambda^{2}$. This allows to choose a typical value for the pion decay constant in the chiral limit, say $f_{\pi, 0}=90 \mathrm{MeV}$, and study the behavior of the chiral condensate as a function of $G \Lambda^{2}$ for various models. We have performed this analysis, considering the range of values for which the different approaches are compatible with the empirical bounds $-\langle\bar{q} q\rangle^{1 / 3} \simeq 200$ to $260 \mathrm{MeV}$ [28, 29]. Our results are displayed in Fig. 2. In the upper panel we show the curves corresponding to covariant models, with different form factors. For comparison, in the lower panel we show the results obtained for the case of instantaneous nonlocal models [21, 30], and for the usual NJL model.

Let us start by analyzing the results in the upper panel of Fig. 2. We observe that in all cases it is possible to choose a value of $G \Lambda^{2}$ so that the corresponding condensate falls within the empirical range. For the case of smooth form factors ( $\mathrm{G}$ and $\mathrm{L} 2)$ we note, however, that condensates in the lower half of the empirical range require quite large values of $G \Lambda^{2}$. An important observation is that when the form factors become sharper (Ln with increasing $\mathrm{n}$ ) the corresponding curves approach the values obtained for the Step form factor. This means that although Eq. (35) includes a term with a derivative of the form factor, which naively should be divergent for $n \rightarrow \infty$, this limit is well defined for $f_{\pi, 0}$.

On the other hand, as it can be seen in the upper panel of Fig. 2, in these models the Step limit does not match the curve obtained for the $4 \mathrm{dNJL}$ model, i.e. the NJL model regularized by a four-dimensional covariant cutoff. This fact can be understood as follows. From Eqs. (15) and (35), the explicit form of the function $\mathcal{F}\left(G \Lambda^{2}\right)$ for a covariant model is given by

$$
\mathcal{F}\left(G \Lambda^{2}\right)=x^{-2 / 3} I_{1}^{1 / 3}(x) I_{2}^{-1 / 2}(x)
$$

where $x=\bar{\sigma} / \Lambda$, and

$$
\begin{aligned}
& I_{1}(x)=\frac{N_{c}}{4 \pi^{2}} \int_{0}^{\infty} d z \frac{z s(z)}{z+x^{2} s^{2}(z)}, \\
& I_{2}(x)=\frac{N_{c}}{8 \pi^{2}} \int_{0}^{\infty} d z \frac{2 z s^{2}(z)-z^{2} s(z) s^{\prime}(z)}{\left[z+x^{2} s^{2}(z)\right]^{2}} .
\end{aligned}
$$

Here we have defined $s(z)=g\left(z \Lambda^{2}\right)=\left[r\left(z \Lambda^{2}\right)\right]^{2}$, whereas $s^{\prime}(z)=d s(z) / d z$. The value of $x$ depends implicitly on the dimensionless parameter $G \Lambda^{2}$ through the gap equation

$$
1=2 G \Lambda^{2} I_{3}(x),
$$

where

$$
I_{3}(x)=\frac{N_{c}}{4 \pi^{2}} \int_{0}^{\infty} d z \frac{z s^{2}(z)}{z+x^{2} s^{2}(z)} .
$$



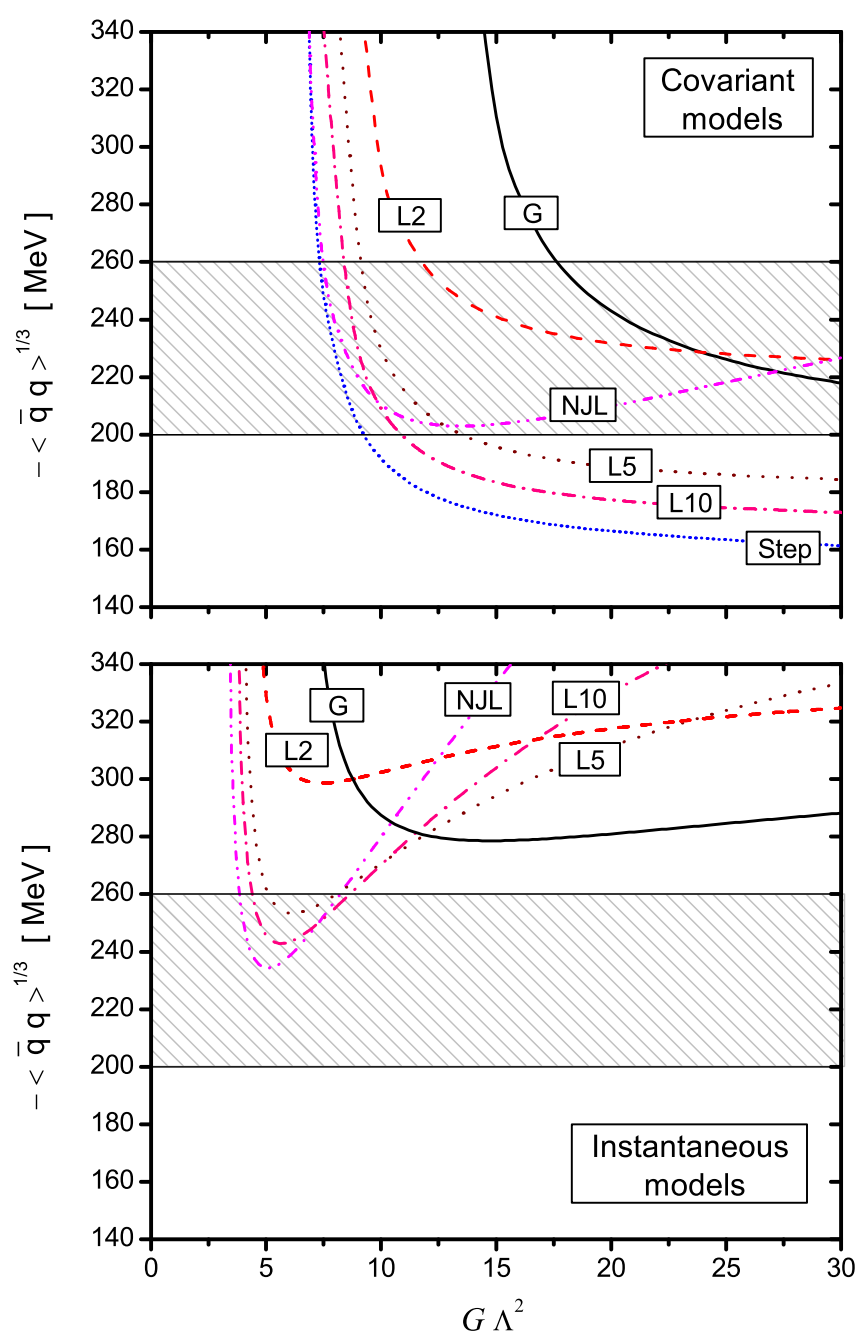

FIG. 2: Chiral condensate as a function of $G \Lambda^{2}$ for different models in the chiral limit. Upper and lower panels correspond to covariant and instantaneous interactions, respectively.

In general, the integrals $I_{i=1,2,3}$ cannot be performed analytically. However, they can be evaluated for the case of a Step form factor by considering the function

$$
s(z)=\left\{\begin{aligned}
1 & \text { for } & z & <1-\alpha / 2 \\
(1+\alpha / 2-z) \alpha^{-1} & \text { for } & 1-\alpha / 2 & <z<1+\alpha / 2 \\
0 & \text { for } & z & >1+\alpha / 2
\end{aligned}\right.
$$

and taking the limit $\alpha \rightarrow 0$. By doing this, we find that in the Step limit the integrals $I_{1}$ and $I_{3}$ are equal to each other, and they are also coincident with their 4dNJL model counterparts (the latter can be found e.g. in Ref. [3]). The actual results are

$$
I_{1}^{\mathrm{Step}}(x)=I_{1}^{4 \mathrm{dNJL}}(x)=I_{3}^{\mathrm{Step}}(x)=I_{3}^{4 \mathrm{dNJL}}(x)=\frac{N_{c}}{4 \pi^{2}}\left[1-x^{2} \ln \left(1+\frac{1}{x^{2}}\right)\right] .
$$

On the other hand, for $I_{2}$ we get

$$
I_{2}^{\mathrm{Step}}(x)=I_{2}^{4 \mathrm{dNJL}}(x)+\Delta I_{2}(x)
$$


where

$$
I_{2}^{4 \mathrm{dNJL}}(x)=\frac{N_{c}}{4 \pi^{2}}\left[\ln \left(1+\frac{1}{x^{2}}\right)-\frac{1}{1+x^{2}}\right], \quad \Delta I_{2}(x)=\frac{N_{c}}{4 \pi^{2}} \frac{1}{4\left(1+x^{2}\right)}
$$

Due to the presence of the $\Delta I_{2}$ contribution, the Step limit of the covariant nonlocal models does not match the 4dNJL result (as shown in Fig. 2). It is not hard to check that $\Delta I_{2}$ comes from the integral of the term with the derivative in Eq. (47), which is nonzero only in the "surface" region $1-\frac{\alpha}{2}<z<1+\frac{\alpha}{2}$. The contribution of this term vanishes in the $4 \mathrm{dNJL}$ according to the usual regularization procedure [3].

It is interesting to compare the results obtained within covariant models with those provided by instantaneous nonlocal models [21, 30]. The latter are shown in the lower panel of Fig. 2. We observe that, in contrast with covariant models, instantaneous nonlocal theories with soft form factors such as G and L2 are not able to yield quark condensates that fall within the empirical range. This can be achieved only for rather sharp form factors, like e.g. L5. Another important difference is that for instantaneous nonlocal models the curves obtained for Ln do converge to the corresponding NJL results (i.e. the results obtained within the NJL model with three-dimensional covariant cutoff, 3dNJL). It is worth to mention, however, that for this type of models $f_{\pi}$ is frame dependent, owing to the noncovariance. Here, to obtain the results displayed in the lower panel of Fig. 2 we have used the pion rest frame, which is the only frame where low energy theorems, such as the GT relation, are fulfilled.

\section{B. Finite current quark mass case}

In the case of finite current quark masses, the model parameters $m_{c}, G$ and $\Lambda$ are usually determined by fitting the pion mass and decay constant to their empirical values $m_{\pi}=139 \mathrm{MeV}$ and $f_{\pi}=92.4 \mathrm{MeV}$, and fixing the chiral condensate at some phenomenologically acceptable value. Since this last quantity is not well determined, we consider here four representative values within the empirical range, namely $(-\langle\bar{q} q\rangle)^{1 / 3}=200,220,240$ and $260 \mathrm{MeV}$. It should be noticed that, in the case of finite $m_{c}$, for schemes I and II one gets different expressions for the integrals in Eqs. (13) and (26), which determine the values of $m_{\pi}$ and $f_{\pi}$. Therefore, the fitted model parameters are also expected to be different in both schemes. Our results are shown in Table I, where we quote the parameter values for schemes I and II, considering different form factors and quark condensates. Interestingly, the current quark mass $m_{c}$ is basically equal for both schemes, while the remaining two parameters $\left(G \Lambda^{2}\right.$ and $\left.\Lambda\right)$ might be rather different, especially in the case of soft form factors and low absolute values of the quark condensate. Perhaps the most striking case corresponds to the L2 form factor: for Scheme II it is not even possible to find a parameter set leading to a condensate of either $-\langle\bar{q} q\rangle^{1 / 3}=200 \mathrm{MeV}$ or $220 \mathrm{MeV}$. For the Gaussian form factor (i.e. the other "soft" function considered here), although we do find compatible parameter sets, the obtained values of $\Lambda$ in Scheme II for the case of low condensates are too low to be reliable for phenomenological applications.

In Table II we quote the values obtained for $\bar{\sigma}$ and $g_{\pi \gamma \gamma}^{2}$, together with the position of the first pole of the quark propagator, for both covariant schemes I and II, and different form factors and quark condensates. As discussed in the literature [16, 17, 18], the absence of purely imaginary poles in the quark propagator can be understood as a realization of quark confinement (we are working here in Euclidean space, thus a purely imaginary pole corresponds to a real pole in Minkowski space). We remark that in the nonlocal covariant models studied here, instead of one single purely imaginary pole - as in the case of the usual NJL model - one has in general an arbitrary number of purely imaginary and/or complex poles [16, 17, 22, 26] (we denote by "complex pole" a pole which has both non-vanishing real and imaginary components). In fact, previous analysis show that for most applications only the first pole, i.e. the closest one to the imaginary axis, is phenomenologically relevant [16, 17, 22, 26]. In the case of Scheme I, as one can see from Table II, among the cases considered the first pole is complex only for a condensate of $200 \mathrm{MeV}$ and form factors G and L10. Moreover, the case of L10 is not phenomenologically viable in view of the low value of $\bar{\sigma}$. Thus, we conclude that if one wants to avoid (following confinement arguments) the presence of low lying purely imaginary poles, the only acceptable situation for Scheme I is that which corresponds to a soft (Gaussian) form factor and a low value of $-\langle\bar{q} q\rangle^{1 / 3}$. If a low lying purely imaginary pole is admitted, then Scheme I with a Gaussian form factor and somewhat larger absolute values of the condensate can be accepted. Sharper form factors would be excluded, leading in all cases to too small values of $\bar{\sigma}$. In the case of Scheme II, one observes that sharp form factors also tend to yield small values of $\bar{\sigma}$, the sole exceptions being the cases L5 and L10 with $-\langle\bar{q} q\rangle^{1 / 3}=200 \mathrm{MeV}$. In these cases, however, the situation is also problematic, since the first poles, although complex, have a very small imaginary part. This implies the existence of a very low $\bar{q} q$ pseudo-threshold, which would require additional prescriptions to preserve meson stability already at energies of a few hundreds of MeV [22]. Given the above discussed limitations of Scheme II for soft form factors and low absolute values of the condensate, we conclude that the sets corresponding to $G$ and L2 form factors and values of $-\langle\bar{q} q\rangle^{1 / 3}$ between 240 and $260 \mathrm{MeV}$ would provide the more acceptable results. Notice 
TABLE I: Model parameters for various models leading to some representative values of the chiral condensate $\left(m_{c}\right.$ and $\Lambda$ given in $\mathrm{MeV}$ )

\begin{tabular}{|c|c|c|c|c|c|c|c|}
\hline \multirow[t]{2}{*}{$-\langle q \bar{q}\rangle^{1 / 3}$} & \multirow[t]{2}{*}{ Form factor } & \multicolumn{3}{|c|}{ Scheme I } & \multicolumn{3}{|c|}{ Scheme II } \\
\hline & & $m_{c}$ & $G \Lambda^{2}$ & $\Lambda$ & $m_{c}$ & $G \Lambda^{2}$ & $\Lambda$ \\
\hline \multirow[t]{4}{*}{200} & G & 9.7 & 18.82 & 651.9 & 9.8 & 71.11 & 459.7 \\
\hline & $\mathrm{L} 2$ & 9.7 & 12.45 & 539.9 & - & - & - \\
\hline & L5 & 9.6 & 8.71 & 799.0 & 9.6 & 13.38 & 660.2 \\
\hline & L10 & 9.8 & 7.57 & 885.0 & 9.7 & 10.68 & 716.5 \\
\hline \multirow[t]{4}{*}{220} & G & 7.4 & 16.98 & 772.0 & 7.4 & 29.06 & 604.0 \\
\hline & L2 & 7.4 & 10.98 & 642.2 & - & - & - \\
\hline & L5 & 7.4 & 8.40 & 924.4 & 7.4 & 10.34 & 790.3 \\
\hline & L10 & 7.5 & 7.47 & 1019.3 & 7.4 & 9.06 & 843.2 \\
\hline \multirow[t]{4}{*}{240} & G & 5.8 & 15.82 & 902.4 & 5.8 & 20.65 & 752.2 \\
\hline & L2 & 5.8 & 10.14 & 751.8 & 5.8 & 16.06 & 586.8 \\
\hline & L5 & 5.8 & 8.20 & 1059.2 & 5.8 & 9.27 & 925.7 \\
\hline & L10 & 5.8 & 7.39 & 1163.0 & 5.8 & 8.36 & 978.6 \\
\hline \multirow[t]{4}{*}{260} & G & 4.6 & 15.08 & 1042.2 & 4.6 & 17.53 & 903.4 \\
\hline & L2 & 4.6 & 9.61 & 868.0 & 4.6 & 11.77 & 736.1 \\
\hline & L5 & 4.6 & 8.07 & 1202.8 & 4.6 & 8.73 & 1067.7 \\
\hline & L10 & 4.6 & 7.34 & 1315.6 & 4.6 & 7.98 & 1122.5 \\
\hline
\end{tabular}

that for these limiting values of the condensate the lowest pole turns out to be complex ("confinement" situation) and purely imaginary, respectively.

Concerning the values obtained for $g_{\pi \gamma \gamma}^{2}$, it is seen that in all cases they are compatible with the experimental range, $g_{\pi \gamma \gamma}^{2}=(7.5 \pm 0.5) \times 10^{-8} \mathrm{MeV}^{-2}$. Now in Table III we quote the parameter values and the results obtained for $\bar{\sigma}$ and $g_{\pi \gamma \gamma}^{2}$ in the $4 \mathrm{dNJL}$ model. We recall that in this case the propagator has a single pole, located at the constituent mass $\Sigma=m_{c}+\bar{\sigma}$ on the imaginary axis. Notice that, as commented in the Introduction, the model does not provide a proper description of the $\pi^{0} \rightarrow \gamma \gamma$ decay width. Phenomenologically acceptable values of $g_{\pi \gamma \gamma}^{2}$ can be obtained instead if the cutoff is (ad-hoc) relaxed to infinity in the triangle loop integrals.

\section{SUMMARY AND CONCLUSIONS}

We have presented a comparative description of chiral quark models which include nonlocal covariant four fermion interactions. These approaches - inspired in features of the underlying QCD theory - represent natural extensions of the usual NJL model and allow to overcome some of its difficulties, in particular those related with the regularization of ultraviolet divergences. We have concentrated here in two alternative ways of introducing the nonlocality, which we have called Scheme I and Scheme II. Using a common notation, we have derived the main expressions to obtain the values of the pion mass, the pion decay constant and some important mean field quantities in terms of the model parameters $m_{c}, G$ and $\Lambda$. In addition, for both schemes we have shown the validity of various well-known relations in the chiral limit.

We have performed numerical calculations for gaussian and lorentzian form factors, studying the behavior of model parameters, constituent masses and propagator poles for given values of the chiral $\bar{q} q$ condensate within a phenomelogically acceptable range. The parameters have been fitted so as to reproduce the empirical values of the pion mass and decay constant. In the chiral limit, the results for covariant nonlocal models have been compared to those obtained within instantaneous nonlocal models. We have found that, in contrast to covariant models, the latter can be hardly accommodated to yield phenomenologically acceptable values of the $\bar{q} q$ condensate. In the case of finite current quark masses, it has been found that sharp form factors such as L5 and L10 are also problematic, leading to either too low values of constituent quark masses or to the appearance of a low $\bar{q} q$ pseudothreshold, which would need additional prescriptions to preserve meson stability. On the other hand, the results seem to favor quark interactions driven by smooth form factors (in our analysis, G and L2). In the case of Scheme II, large values of $-\langle\bar{q} q\rangle$ are found to be preferred, since lower condensates lead to too low values of the "cutoff" $\Lambda$. For Scheme I, instead, lower absolute 
TABLE II: Values for $\bar{\sigma}$ and $g_{\pi \gamma \gamma}^{2}$ and location of the first pole of the quark propagator for various models $\left(g_{\pi \gamma \gamma}^{2}\right.$ given in $\mathrm{MeV}^{-2} \times 10^{-8}$, other quantities given in $\mathrm{MeV}$ )

\begin{tabular}{|c|c|c|c|c|c|c|c|}
\hline \multirow[t]{2}{*}{$-\langle q \bar{q}\rangle^{1 / 3}$} & \multirow[t]{2}{*}{ Form factor } & \multicolumn{3}{|c|}{ Scheme I } & \multicolumn{3}{|c|}{ Scheme II } \\
\hline & & $\bar{\sigma}$ & First pole & $g_{\pi \gamma \gamma}^{2}$ & $\bar{\sigma}$ & First pole & $g_{\pi \gamma \gamma}^{2}$ \\
\hline \multirow[t]{4}{*}{200} & $\mathrm{G}$ & 318 & $180+454 \mathrm{i}$ & 7.43 & 1356 & $499+241 \mathrm{i}$ & 7.16 \\
\hline & $\mathrm{L} 2$ & 296 & $284 \mathrm{i}$ & 7.37 & - & - & - \\
\hline & L5 & 181 & $191 \mathrm{i}$ & 7.57 & 366 & $640+169 i$ & 7.37 \\
\hline & L10 & 149 & $875+131 \mathrm{i}$ & 7.39 & 302 & $711+98 \mathrm{i}$ & 7.24 \\
\hline \multirow[t]{4}{*}{220} & G & 282 & $356 \mathrm{i}$ & 7.50 & 620 & $421+372 \mathrm{i}$ & 7.45 \\
\hline & L2 & 259 & $259 \mathrm{i}$ & 7.49 & - & - & - \\
\hline & L5 & 173 & $180 \mathrm{i}$ & 7.74 & 276 & $757+217 \mathrm{i}$ & 7.45 \\
\hline & L10 & 145 & $152 \mathrm{i}$ & 7.64 & 248 & $835+120 \mathrm{i}$ & 7.46 \\
\hline \multirow[t]{4}{*}{240} & G & 255 & $288 \mathrm{i}$ & 7.57 & 424 & $288+510 \mathrm{i}$ & 7.49 \\
\hline & $\mathrm{L} 2$ & 233 & $237 \mathrm{i}$ & 7.55 & 475 & $485+304 i$ & 7.48 \\
\hline & L5 & 166 & $153 \mathrm{i}$ & 7.87 & 238 & $243 \mathrm{i}$ & 7.52 \\
\hline & L10 & 142 & $147 \mathrm{i}$ & 7.96 & 221 & $968+142 \mathrm{i}$ & 7.57 \\
\hline \multirow[t]{4}{*}{260} & $\mathrm{G}$ & 235 & $255 \mathrm{i}$ & 7.61 & 339 & $430 \mathrm{i}$ & 7.52 \\
\hline & $\mathrm{L} 2$ & 216 & $219 \mathrm{i}$ & 7.65 & 330 & $323 \mathrm{i}$ & 7.50 \\
\hline & L5 & 160 & $172 \mathrm{i}$ & 7.94 & 215 & $220 \mathrm{i}$ & 7.61 \\
\hline & L10 & 139 & $143 \mathrm{i}$ & 8.11 & 204 & $1110+165 \mathrm{i}$ & 7.66 \\
\hline
\end{tabular}

TABLE III: Model parameters and values of $\bar{\sigma}$ and $g_{\pi \gamma \gamma}^{2}$ for representative values of the chiral condensate in the $4 \mathrm{dNJL}$ model $\left(g_{\pi \gamma \gamma}^{2}\right.$ given in $\mathrm{MeV}^{-2} \times 10^{-8}, m_{c}, \Lambda$ and $\bar{\sigma}$ given in $\left.\mathrm{MeV}\right)$

\begin{tabular}{cccccc}
$-\langle q \bar{q}\rangle^{1 / 3}$ & $m_{c}$ & $G \Lambda^{2}$ & $\Lambda$ & $\bar{\sigma}$ & $g_{\pi \gamma \gamma}^{2}$ \\
\hline 200 & - & - & - & - & - \\
\hline 220 & 7.7 & 9.13 & 807.8 & 297.9 & 4.40 \\
\hline 240 & 6.0 & 7.93 & 947.8 & 244.1 & 5.79 \\
\hline 260 & 4.7 & 7.43 & 1094.6 & 218.2 & 6.50 \\
\hline
\end{tabular}

values of the condensate seem to be favored in order to avoid relatively small constituent quark masses. As it is shown in Table II, in some of these cases one finds that the first pole of the quark propagator has both a real and an imaginary part, which can be understood as a manifestation of confinement. This pole structure is a characteristic of the form factor under consideration, and can be extended to more involved approaches such as e.g. the instanton liquid model. Finally, we have considered the case of the NJL model regularized by a four dimensional cutoff (4dNJL), analyzing its deviation from a nonlocal model with a step-like form factor.

\section{Acknowledgments}

The authors are glad to thank D. Aguilera, D. Blaschke and H. Grigorian for useful comments and discussions. This work has been supported in part by CONICET and ANPCyT (Argentina), under grants PIP 02368, PIP 6084, PIP 6009, PICT00-03-08580 and PICT02-03-10718.

[1] Y. Nambu and G. Jona-Lasinio, Phys. Rev. 122, 345 (1961); Phys. Rev. 124, 246 (1961).

[2] U. Vogl and W. Weise, Prog. Part. Nucl. Phys. 27, 195 (1991).

[3] S. P. Klevansky, Rev. Mod. Phys. 64, 649 (1992). 
[4] T. Hatsuda and T. Kunihiro, Phys. Rept. 247, 221 (1994).

[5] J. Bijnens, E. de Rafael and H. q. Zheng, Z. Phys. C 62, 437 (1994); J. Bijnens and J. Prades, Phys. Lett. B 320, 130 (1994); J. Bijnens and J. Prades, Z. Phys. C 64, 475 (1994).

[6] T. Meissner, E. Ruiz Arriola and K. Goeke, Z. Phys. A 336, 91 (1990).

[7] V. Dmitrasinovic, H. J. Schulze, R. Tegen and R. H. Lemmer, Annals Phys. 238, 332 (1995); E. N. Nikolov, W. Broniowski, C. V. Christov, G. Ripka and K. Goeke, Nucl. Phys. A 608, 411 (1996);

[8] C. Schuren, F. Doring, E. Ruiz Arriola and K. Goeke, Nucl. Phys. A 565, 687 (1993).

[9] G. Ripka, Quarks bound by chiral fields (Oxford University Press, Oxford, 1997).

[10] T. Schafer and E. V. Shuryak, Rev. Mod. Phys. 70, 323 (1998).

[11] C. D. Roberts and A. G. Williams, Prog. Part. Nucl. Phys. 33, 477 (1994); C. D. Roberts and S. M. Schmidt, Prog. Part. Nucl. Phys. 45, S1 (2000).

[12] M. B. Parappilly, P. O. Bowman, U. M. Heller, D. B. Leinweber, A. G. Williams and J. B. Zhang, Phys. Rev. D 73, 054504 (2006).

[13] E. Ruiz Arriola and L. L. Salcedo, Phys. Lett. B 450, 225 (1999).

[14] D. Blaschke, Y. L. Kalinovsky, G. Roepke, S. M. Schmidt and M. K. Volkov, Phys. Rev. C 53, 2394 (1996).

[15] G. Ripka, Nucl. Phys. A 683, 463 (2001); R. S. Plant and M. C. Birse, Nucl. Phys. A 703, 717 (2002).

[16] R. D. Bowler and M. C. Birse, Nucl. Phys. A 582, 655 (1995).

[17] R. S. Plant and M. C. Birse, Nucl. Phys. A 628, 607 (1998).

[18] M. Stingl, Phys. Rev. D 34, 3863 (1986) [Erratum-ibid. D 36, 651 (1987)]; H. J. Munczek, Phys. Lett. B 175, 215 (1986); C. J. Burden, C. D. Roberts and A. G. Williams, Phys. Lett. B 285, 347 (1992); G. Krein, C. D. Roberts and A. G. Williams, Int. J. Mod. Phys. A 7, 5607 (1992); D. Blaschke, G. Burau, Y. L. Kalinovsky, P. Maris and P. C. Tandy, Int. J. Mod. Phys. A 16, 2267 (2001).

[19] M. Buballa and S. Krewald, Phys. Lett. B 29419 (1992).

[20] H. Ito, W. Buck and F. Gross, Phys. Rev. C 43 (1991) 2483; H. Ito, W. W. Buck and F. Gross, Phys. Rev. C 451918 (1992).

[21] S. M. Schmidt, D. Blaschke and Y. L. Kalinovsky, Phys. Rev. C 50435 (1994).

[22] A. Scarpettini, D. Gomez Dumm and N. N. Scoccola, Phys. Rev. D 69, 114018 (2004).

[23] S. Noguera, arXiv:hep-ph/0502171 S. Noguera and V. Vento, arXiv:hep-ph/0505102

[24] B. Golli, W. Broniowski and G. Ripka, Phys. Lett. B 437, 24 (1998); W. Broniowski, B. Golli and G. Ripka, Nucl. Phys. A 703, 667 (2002).

[25] A. H. Rezaeian, N. R. Walet and M. C. Birse, Phys. Rev. C 70, 065203 (2004); A. H. Rezaeian and H. J. Pirner, Nucl. Phys. A 769, 35 (2006).

[26] I. General, D. Gomez Dumm and N. N. Scoccola, Phys. Lett. B 506, 267 (2001); D. Gomez Dumm and N. N. Scoccola, Phys. Rev. D 65, 074021 (2002); Phys. Rev. C 72, 014909 (2005); R. S. Duhau, A. G. Grunfeld and N. N. Scoccola, Phys. Rev. D 70, 074026 (2004); D. Gomez Dumm, D. B. Blaschke, A. G. Grunfeld and N. N. Scoccola, Phys. Rev. D 73, 114019 (2006).

[27] J. W. Bos, J. H. Koch and H. W. L. Naus, Phys. Rev. C 44, 485 (1991).

[28] H. G. Dosch and S. Narison, Phys. Lett. B 417, 173 (1998).

[29] L. Giusti, F. Rapuano, M. Talevi and A. Vladikas, Nucl. Phys. B 538, 249 (1999).

[30] H. Grigorian, arXiv:hep-ph/0602238 